Chapman University

Chapman University Digital Commons

Physical Therapy Faculty Articles and Research

Physical Therapy

2009

\title{
Nonlinear Time Series Analysis Of Knee and Ankle Kinematics During Side By Side Treadmill Walking
}

Jeff A. Nessler

California State University - San Marcos

Charles J. De Leone

California State University - San Marcos

Sarah Gilliland

Chapman University, sgillila@chapman.edu

Follow this and additional works at: http://digitalcommons.chapman.edu/pt_articles

Part of the Physical Therapy Commons

\section{Recommended Citation}

Nessler, Jeff A., Charles J. De Leone, and Sara Gilliland. (2009). "Nonlinear time series analysis of knee and ankle kinematics during side by side treadmill walking." Chaos: An Interdisciplinary Journal of Nonlinear Science 19(2), 026104.

DOI: $10.1063 / 1.3125762$ 


\section{Nonlinear Time Series Analysis Of Knee and Ankle Kinematics During Side By Side Treadmill Walking}

\section{Comments}

This article was originally published in Chaos: An Interdisciplinary Journal of Nonlinear Science, volume 19, issue 2, in 2009. doi: DOI: 10.1063/1.3125762

\section{Copyright}

American Institute of Physics 


\title{
Nonlinear time series analysis of knee and ankle kinematics during side by side treadmill walking
}

\author{
Jeff A. Nessler, ${ }^{1, a)}$ Charles J. De Leone, ${ }^{2, b)}$ and Sara Gilliland ${ }^{3, c)}$ \\ ${ }^{1}$ Department of Kinesiology, California State University, San Marcos, California 92096, USA \\ ${ }^{2}$ Department of Physics, California State University, San Marcos, California 92096, USA \\ ${ }^{3}$ Department of Physical Therapy, Chapman University, Orange, California 92866, USA
}

(Received 11 February 2009; accepted 7 April 2009; published online 29 June 2009)

\begin{abstract}
Nonlinear time series analysis was used to estimate maximal Lyapunov exponents of select ankle and knee kinematics during three different conditions of treadmill walking: independent, side by side, and side by side with forced synchronization of stepping. Stride to stride variability was significantly increased for the condition in which individuals walked side by side and synchronized unintentionally when compared to the conditions of forced synchronization and independent walking. In addition, standard deviations of three kinematic variables of lower extremity movement were significantly increased during the condition in which unintentional synchronization occurred. No relationship was found between standard deviation and estimates of maximal Lyapunov exponents. An increase in kinematic variability during side by side walking for nonimpaired individuals who are not at risk of falling suggests that variability in certain aspects of performance might be indicative of a healthy system. Modeling this variability for an impaired individual to imitate may have beneficial effects on locomotor function. These results may therefore have implications for the rehabilitation of gait in humans by suggesting that a different functional outcome might be achieved by practicing side by side walking as opposed to more commonly used strategies involving independent walking. (C) 2009 American Institute of Physics. [DOI: 10.1063/1.3125762]
\end{abstract}

Unintentional synchronization of movement has been observed to occur between humans during several activities, including side by side walking. Tracking the movements of one's partner might involve the activity of mirrorneuron networks thought to contribute to action imitation, an important facet of skill acquisition. This inherent tendency to synchronize might be useful in the rehabilitation of gait following neurological impairment by providing a means to relearn proper lower-limb movement patterns. To evaluate the effects of side by side walking in healthy individuals, nonlinear time series analysis techniques were combined with traditional gait analysis to evaluate six measures of ankle and knee kinematics of subjects walking on a motorized treadmill under three different conditions: independent walking, walking next to a partner in which unintentional synchronization occurred, and walking next to a partner while intentionally synchronizing. The results indicated that walking with unintentional synchronization to one's partner significantly altered the lower-limb stride to stride dynamics in each individual when compared with either the independent walking condition or the intentional synchronization condition. These results suggest that uninstructed side by side walking causes an individual to walk in a manner that is more variable than their normal stepping pattern.

\footnotetext{
a) Telephone: (760) 750-7352. Fax: (760) 750-3190. Electronic mail: jnessler@csusm.edu.

${ }^{b)}$ Telephone: (760) 750-8074. Fax: (760) 750-3190. Electronic mail: cdeleone@csusm.edu.

c) Telephone: (714) 744-7620. Fax: (714) 744-7621. Electronic mail: gilli104@chapman.edu.
}

Increased variability in lower-limb joint angles during walking might be desirable in certain situations by adding adaptability to movement. This adaptability might be modeled by a healthy system during side by side walking with an impaired individual. Additional research is needed to understand the long term effects of this behavior and its potential utility as a therapeutic exercise.

\section{INTRODUCTION}

Humans demonstrate a tendency to synchronize movement to external, rhythmic cues, often unintentionally. ${ }^{1-4}$ This behavior has been shown to occur during bipedal gait, where it can be intentional and voluntary ${ }^{5-7}$ or unintentional and involuntary. ${ }^{8-11}$ In previous work, intentional synchronization has received greater interest, possibly because it is easier to reproduce and manipulate in a laboratory or clinic and may therefore be applied more readily to rehabilitation of gait following neurological impairment. For example, previous investigators have successfully used rhythmic auditory cues to improve the stepping of patients with Parkinson disease (e.g., Refs. 5 and 12) and poststroke. ${ }^{6}$ However, forced entrainment to a constant, repetitive rhythm may not be the optimal way to exploit this phenomenon for the purpose of restoring function to gait. Hausdorff et al. ${ }^{7,13}$ demonstrated that when healthy individuals were constrained to walk in rhythm with a metronome, a decrease in the stability of long range correlations in stride time occurred, similar to that observed in diseased individuals. While increases in stride time variability have been related to gait pathology in multiple populations, ${ }^{12-14}$ normal variations in stride time persist in 
healthy individuals that a metronome is unable to reproduce. In other biological processes, a decrease in the variability of a system's output can be indicative of pathology, ${ }^{15-17}$ suggesting that rigid constraint of a system's output in some cases may lead to decreased function.

Synchronization of movement may be more effective for gait rehabilitation if the external cue exhibits a variation that is consistent with a healthy physiological system. In addition, increased motor skill is typically associated with less intentional control over a movement (i.e., passive control), suggesting that a situation utilizing subconscious or unintentional synchronization may be preferable. ${ }^{18-20}$ Side by side walking might be a means to achieve a healthy biological signal for synchronization and is a situation in which interpersonal entrainment is frequently observed to occur naturally and unintentionally. ${ }^{8,911,21}$ This unintentional phenomenon likely involves a type of mirror-neuron network, whereby the simple perception of the movements of one's partner via visual (e.g., Ref. 22), auditory, ${ }^{23}$ or mechanical pathways will induce activity in neurons that are also active when an individual carries out the movement themselves. Previous research has demonstrated that such networks play a significant role in action imitation, which may be fundamental to learning or relearning a motor skill (for review, see Ref. 24). The idea of action imitation can also be found in the study of social dynamics, where an individual's movement pattern has been described as the result of an interaction between their preferred movement pattern and that of surrounding individuals. ${ }^{25,26}$ In the case where individuals walk side by side, both systems experience an attraction to adapt their output to imitate their partner's movements, leading to a mutual cadence that is the result of a negotiation between their preferred pattern and that of their partner. Coupling an impaired individual with a healthy individual might therefore provide a synchronizing signal that exhibits appropriate variations in movement patterns and encourages the impaired individual to imitate and adopt these patterns in a subconscious manner. An improved understanding of this phenomenon may provide additional insight to the control of bipedal locomotion and may help to inform therapeutic techniques that involve synchronization for the purpose of altering step kinematics in a rehabilitation setting.

Nonlinear time series analysis is a useful approach for elucidating subtle differences in the dynamics of locomotion for normal walking and unintentional and intentional synchronization. Specifically, variability in the movement kinematics of gait (i.e., knee or ankle angle, step height, etc.) has received increasing interest of late, and a nonlinear approach to the analysis of variability is attractive because it can provide insight that traditional gait analysis techniques cannot. ${ }^{27-30}$ For example, utilizing standard deviation to evaluate the variability of kinematic time series data ignores the time dependent attributes of variability by assuming that each step is independent and unrelated to previous steps and that stride to stride variations are random. A more detailed analysis of stride to stride variability during gait can be achieved over the relatively short term (zero to ten strides ${ }^{27-29}$ ) with the estimation of maximal Lyapunov exponents. In this context, greater values for maximal Lyapunov exponents indicate that an individual has greater stride to stride variability and is less periodic over the time interval studied, and lower values indicate the opposite. This type of analysis can yield information regarding the nature of neurological control by quantifying the ability of the system to respond to and assimilate minor mechanical and physiological perturbations to the intended movement pattern. ${ }^{27-29,31,32}$ While there is currently debate over the chaotic nature of bipedal locomotion, ${ }^{27,29}$ studying stride to stride variability of gait kinematics using maximal Lyapunov exponents has still provided meaningful information. For example, several previous investigators have utilized a similar analysis to apply the idea of kinematic variability to the prediction of fall risk in the elderly. ${ }^{14,28,29,33}$

Overall, the relationship between kinematic variability during gait and the health of the locomotor system is not entirely clear. For example, there is ample evidence that increased variability in stride time is related to pathology and an increased risk of fall in both elderly and diseased populations. $^{7,13,14}$ In addition, increases in maximal Lyapunov exponents for lower-limb kinematic variables have been associated with increased age. ${ }^{28}$ However, too little variability may also be problematic, as decreases in stride to stride variability in joint angle kinematics have also been associated with gait pathology. ${ }^{34-36}$ These data suggest that some variability might be beneficial to walking stability and indicative of a healthy locomotor system. ${ }^{37,38}$ Further support for this idea can be found in passive dynamic walking models, where increasing the flexibility in a walker's movement pattern will allow it to avoid falling under increasing variations in initial conditions. ${ }^{30,39} \mathrm{~A}$ wider basin of attraction for locomotion in these simplified models corresponds to additional movement patterns from which to select in response to a perturbation. In humans, an increased "library" of stable gait patterns may lead to increases in variability under conditions in which overground stability is challenged. In this context, an individual with fewer alternatives for movement patterns that will allow them to maintain balance in response to perturbation will likely demonstrate decreased movement variability and an increased risk of falling.

Optimizing stride to stride, lower-limb movement variability may therefore be a worthwhile goal in the restoration of locomotor function. It is possible that such a task might be achieved through uninstructed side by side treadmill walking by stimulating mirror-neuron networks in a manner that encourages synchronization and interferes with an individual's preferred walking pattern. In this situation, a healthy and flexible system would likely utilize additional lower-limb movement patterns to maintain overground stability while matching their partner's movements. Practicing walking side by side might therefore be a means to increase healthy variability by challenging an impaired individual to recruit additional movement patterns in order to avoid falling. For individuals with excessive kinematic variability, this strategy may help to improve function by providing a template of a locomotor system that exhibits a healthy combination of both stride time consistency and joint kinematic variation, which might be successfully imitated and learned. 
The purpose of this study was to use the techniques of nonlinear time series analysis and traditional gait analysis to examine the variability of multiple kinematic variables of bipedal locomotion under varying conditions of synchronization. This analysis is designed to serve as a first step in the analysis of differences in the dynamics of normal, unintentional, and intentional synchronization. Because previous data have indicated that individuals walking side by side will alter their movement patterns, ${ }^{11,21}$ it was hypothesized that differences in variability would arise among the three conditions studied, with the least amount of locomotor variability found during independent walking. If present, differences among these three conditions would suggest that an increase in movement variability during gait can be seen in healthy individuals not at risk of falling, suggesting that an increase in flexibility of certain aspects of locomotion might be a hallmark of a healthy system. These results may also provide insight to the use of side by side treadmill walking as a therapeutic exercise in the rehabilitation of gait for impaired patients by demonstrating whether movement variability during gait can be selectively altered in this manner. Since most commonly used therapeutic techniques for gait rehabilitation involve independent walking, a difference in stride to stride variability of gait would suggest the possibility of different outcomes for exercises involving side by side walking.

\section{METHODS}

\section{A. Subjects}

Fourteen subjects were recruited from the local student population $($ age $=23.33 \pm 5.06$ years, height $=174.33 \pm 9.94 \mathrm{~cm}$, mass $=73.65 \pm 19.76 \mathrm{~kg}$ ). Nine male and five female subjects participated in the study, resulting in four male-male pairings, two female-female pairings, and one mixed pairing. All subjects demonstrated normal gait, free of neurological impairment, and musculoskeletal injury. Some effort was made to combine subjects with similar leg lengths, as it was previously noted that differences in leg length contribute to a decrease in the amount of unintentional synchronization. ${ }^{11,21}$ However, most pairings exhibited some difference in leg length, with an overall mean difference of $6.49 \pm 5.25 \mathrm{~cm}$. All procedures were approved by the Institutional Review Board, and all participants gave their informed consent prior to data collection. All subjects were kept naive as to the purpose of the experiment until data collection had ended.

\section{B. General procedures}

This study was a repeated measures design with each subject walking under three different conditions. For all trials, subjects walked on a motorized treadmill (Vision Fitness T9800S) at $2.5 \mathrm{mph}(4.02 \mathrm{~km} / \mathrm{h})$ and were given a $2 \mathrm{~min}$ warm-up period immediately prior to data collection. Treadmills were modified by removing the hand rails and placing the instrument panel on the floor so that there were no obstructions to camera view or the subject's view of their partner. For the first condition (solo), subjects walked by themselves for a period of $2.5 \mathrm{~min}$. During this time, subjects were instructed to walk as normally as possible, with no restriction placed on speech or direction of gaze. For the second condition (paired), subjects walked on the same treadmill, while a partner walked on a similar, adjacent treadmill at the same speed for a period of $5 \mathrm{~min}$. Again, subjects were asked to walk as normally as possible, and no restriction was given to talking or the focus of their vision. For the third condition (forced), subjects walked in pairs but were instructed to purposely synchronize their stepping, such that each step was coupled in phase, similar to marching in step. The duration of the third trial was $2.5 \mathrm{~min}$. All trials took place in this order so as to minimize the possibility that the forced condition would affect performance of subjects during the paired condition by prematurely drawing attention to their partner's steps.

Previous data indicate that during a typical trial in which individuals are expected to synchronize unintentionally, subjects will entrain for only a portion of the trial, and this percentage varies greatly among pairings. ${ }^{11,21}$ Therefore, subjects walked for a longer period of time under the paired condition to provide additional opportunity to synchronize. This approach allowed for the objective selection of a period of time in which the greatest amount of frequency locking occurred, thereby ensuring a larger amount of unintentional synchronization over the period analyzed without directly influencing the stepping behavior of the subjects.

For all trials, sagittal plane kinematic data were collected at $120 \mathrm{~Hz}$ using a six camera optical motion capture system (Vicon MX3+). Markers were placed over the toe, heel, lateral maleolus, lateral knee, and lateral thigh of the right leg of both subjects. From these markers, measures of knee angle, ankle angle, ankle $X$ trajectory (step length), ankle $Y$ trajectory (step height), ankle $Z$ trajectory (frontal plane movement), and knee $Y$ trajectory were calculated. Standard kinematic analysis focused on knee angle, ankle angle, step height, and step length. For these variables, one stride was defined from one local minima of ankle $X$ data to the data point just before the next local minima. Each ankle $X$ local minima represents the point at which the ankle is furthest posterior for a particular stride, just before toe off.

\section{Data analysis}

Data analysis involved the use of custom MATLAB routines, combined with the free TISEAN software package [Version 3.0.1 (Ref. 40)] and additional free software developed by Perc. ${ }^{41}$ In MATLAB, raw marker data were filtered (fourth order Butterworth, cutoff $=100 \mathrm{~Hz}$ ), and truncated into 2 min trials. In addition, all data were normalized in time with respect to the overall mean cadence of the sample $(n=14)$ during solo walking. For the solo and forced conditions, the first $30 \mathrm{~s}$ of data (approximately 26 steps) were excluded from the analysis to generate the $2 \mathrm{~min}$ trial. For the paired condition (condition 2), a continuous 2 min window was found in which the greatest amount of step frequency matching occurred between subjects within the original 5 min trial. Average step frequency for each subject was calculated using a moving $5 \mathrm{~s}$ window across the entire trial. Frequency locking was defined for a particular data point if the difference in step frequency was less than $0.02 \mathrm{~Hz}$ between subjects. This 


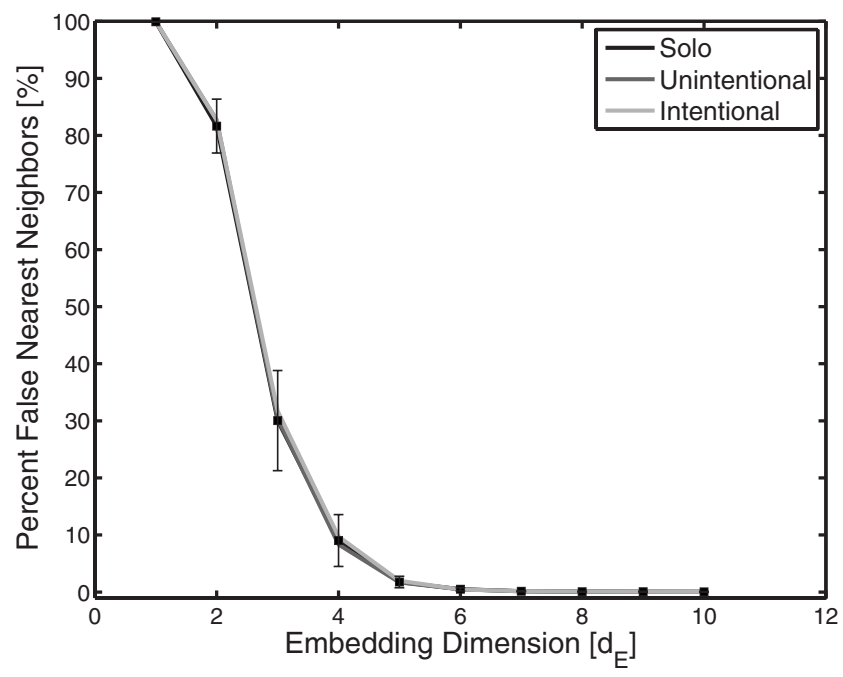

FIG. 1. False nearest neighbor results for knee angle data over three different walking conditions ( $n=14$ for each condition). These results were typical for all of the variables examined. Based on this analysis, an embedding dimension of 6 was selected, although previous investigators have typically selected an embedding dimension of 5 .

procedure is a modified version of the one described in Ref. 9. Of the $2 \mathrm{~min}$ windows selected for each trial of unintentional entrainment, the average amount of time in which subjects were synchronized was $73.2 \pm 26.8 \mathrm{~s}$. During the forced condition, subjects were entrained for $99.27 \pm 0.01 \%$ of the trial, similar to that observed in previous experiments. ${ }^{11}$ Each data set was then $2 \mathrm{~min}$ in length (14 400 data points) and encompassed an average of $103 \pm 6.38$ strides.

Nonlinear time series analysis was used to determine stride to stride variability of six kinematic variables of gait for each trial (ankle angle, ankle $X$, ankle $Y$, ankle $Z$, knee angle, knee $Y$ ). This analysis began with the reconstruction of the state space to allow for a concise definition of the system at each point. Each state space was represented by

$$
X(t)=\left[x(t), x(t+\tau), \ldots, x\left(1+\left(d_{E}-1\right) \tau\right)\right],
$$

where $X(t)$ represents the new state vector of dimension $d_{E}$, which retains all of the properties of the original time series, $x(t)$, and has as its time delay $\tau$, and $d_{E}$ as its embedding dimension. Beginning with the original time series, the embedding delay was determined by finding the first minima of the average mutual information algorithm described by Refs. 41-43. This approach seeks to minimize the amount of redundant information among data sets that span multiple time delays. Next, the optimal embedding dimension was calculated using the false nearest neighbor algorithm. ${ }^{44} \mathrm{Al}-$ though previous investigators have utilized an embedding dimension of 5 when analyzing sagittal plane gait kinematics, ${ }^{27,28,41}$ we found that on average, the percent of false nearest neighbors did not completely converge to zero until a dimension of 6 was used (Fig. 1). This result was consistent across all subjects and all variables. Both the average mutual information and false nearest neighbor calculations were performed using software designed by Perc. ${ }^{41}$

Stationarity and determinism were evaluated for each data set first by generating and inspecting recurrence plots. ${ }^{45}$
Recurrence plots are graphical representations of the Euclidean difference between all points within a specified radius in a state space. The plot is created when the differences between points $X(i)$ and $X(j)$ are plotted in the $(i, j)$ plane. Large inconsistencies in the texture of the recurrence plot indicate regions of nonstationarity. The presence of continuous lines can be interpreted as regions of periodic behavior, thus providing a gross method of evaluating determinism. Determinism was also evaluated using the method first described by Kaplan and Glass, ${ }^{46}$ implemented in software by Perc. $^{41}$

Following reconstruction of the state space, divergence curves and maximal Lyapunov exponents $(\lambda)$ were calculated for each data set. The maximal Lyapunov exponent for a particular time series can be obtained from the equation

$$
d(t)=d_{0} e^{\lambda t},
$$

where $d(t)$ is the Euclidean distance or amount of divergence at time $t$ and $d_{0}$ is the initial distance between the neighboring trajectories. In Eq. (2), finding $\lambda$ over an interval of time provides a measure of the rate of divergence of trajectories with small initial Euclidean differences that then increase as time is incremented forward. Maximal Lyapunov exponents therefore provide a measure of the sensitivity of a system to minute perturbations, such as the small stride to stride fluctuations in movement dynamics that occur naturally during normal walking. It should be noted that Eq. (2) defines true Lyapunov exponents, which arise from the limits as $t \rightarrow \infty$ and as $d_{0} \rightarrow 0$. Estimates of finite-time Lyapunov exponents $\left(\lambda^{*}\right)$ are typically used in the analysis of discrete time series data, and multiple approaches have been described previously. ${ }^{47,48}$ In particular, the method described by Rosenstein et al. ${ }^{48}$ is defined as

$$
\ln \left[d_{j}(i)\right] \approx \ln C_{j}+\lambda^{*}(i \cdot \Delta t),
$$

where for the $j$ th pair of nearest neighbors, $d_{j}(i)$ refers to the Euclidean distance following $i$ discrete time steps, and $C_{j}$ refers to the initial Euclidean distance. In order to estimate $\lambda^{*}$ over all pairs of nearest neighbors in a time series, a mean divergence curve $y$ can be calculated from the equation

$$
y(i)=(1 / \Delta t)\left\langle\ln \left[d_{j}(i)\right]\right\rangle,
$$

where $\langle\ldots\rangle$ denotes the average over all $j$ pairs of nearest neighbors. Using the free TISEAN software package, ${ }^{40}$ mean divergence curves were calculated for the present data for each time series based on this approach. To minimize any potential effects arising from temporal correlations among successive elements in a time series, a time interval was defined about each discrete data point where nearest neighbors were ignored, known as Theiler window $(w) .{ }^{49,50}$ For the present analysis, $w$ was set to approximately six strides (800 data points).

Maximal Lyaponov exponents were calculated for two different regions of the divergence curves to yield an estimate of variability over $0-1$ stride ("short term," $\lambda_{\text {short }}$ ) and 4-10 strides ("long term," $\lambda_{\text {long }}$ ) (Fig. 2). The practice of examining variability over these intervals has been utilized in previous analyses of gait data. ${ }^{29,31,51}$ This procedure was selected for the present analysis because previous investiga- 


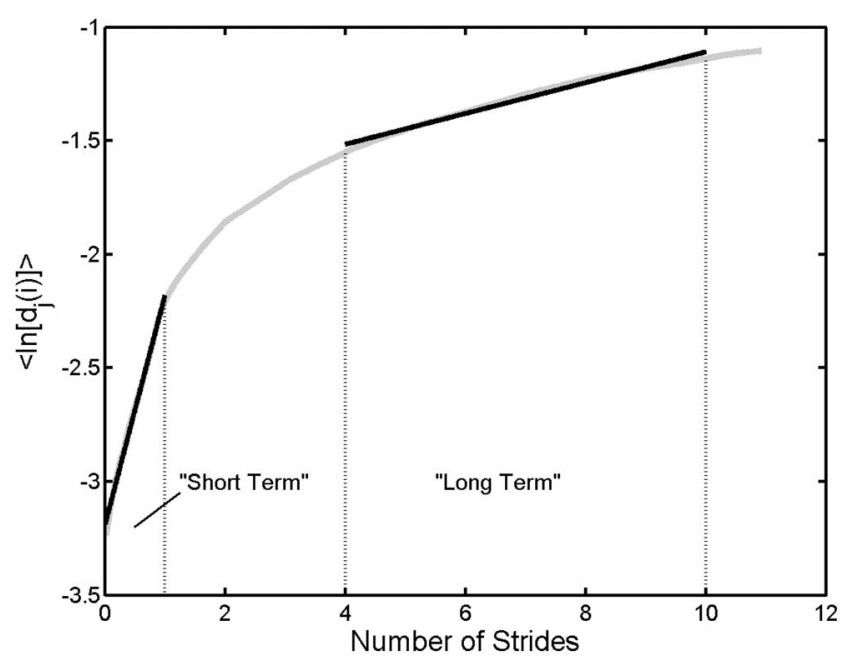

FIG. 2. Graphical description of the procedure for estimating short term and long term maximal Lyapunov exponents. $\lambda_{\text {short }}$ and $\lambda_{\text {long }}$ were determined by fitting a least-squares linear regression to the divergence curve over $0-1$ and 4-10 strides, respectively, and then calculating the slope of each line.

tors have reported different values in their estimation of maximal Lyapunov exponents for various aspects of gait analysis, presumably due to differences in the interval over which the slope of the divergence curve was taken. Here, a relatively well established approach was undertaken by estimating slopes of the divergence curves by fitting a line to both of these regions using least-squares regression.

Positive maximal Lyapunov exponents, together with evidence of determinism, are characteristics of a chaotic system. ${ }^{43,52}$ However, random data can also yield positive Lyapunov exponents, and analysis of surrogate data is useful to distinguish a deterministic system from one with stochastic noise. Here, surrogate data were generated with the TISEAN software, implementing a phase randomization technique for each solo walking data set. Estimates of "short" and "long" term maximal Lyapunov exponents were then obtained for the surrogate data in the manner described above and compared with those obtained from the original time series data.

Finally, multivariate repeated measure analysis of variance was used to determine if estimates of maximal Lyapunov exponents and standard kinematic measures were significantly different among the three gait conditions and variables analyzed. A significant result was followed up with repeated measures ANOVA for each variable, followed by individual paired t-tests to determine differences among gait conditions. A familywise alpha $=0.05$ (Bonferroni posthoc adjustment) was used for multiple comparisons within each repeated measures ANOVA.

\section{RESULTS}

The statistical omnibus test (multivariate repeated measures ANOVA) indicated the presence of a significant difference across the three walking conditions for all variables tested $(p<0.001)$. The mean and standard deviations for four conventional measures of gait are presented in Table I. During analysis, it was noted that since subjects negotiated a mutual cadence during synchronization, it was typical for one partner to increase a particular variable, such as step length, and the other partner to decrease the same variable. As a result, it is unlikely that changes in kinematic parameters would be reflected in the overall mean value for each parameter studied. This observation was demonstrated in the present results, as mean values changed very little across conditions for any of the variables examined. However, variability of gait (i.e., standard deviation) increased significantly during the paired condition when compared to the solo condition for knee angle $(p<0.001)$ and step length $(p$ $<0.001)$. In addition, ankle angle standard deviation was not significantly different across conditions when all subjects were analyzed, but subsequent analysis following the removal of outlier data for one subject did yield a significant difference between the solo and paired conditions for this variable $(p=0.009)$. Standard deviation also increased significantly during the forced condition when compared with the solo condition for step length $(p=0.006)$.

Nonlinear time series analysis was initially planned to include ankle movements in the $X$ (step length) and $Z$ (sway in the frontal plane) directions, in addition to the variables presented in Table II. However, upon inspection of the recurrence plots for both of these variables, substantial nonstationarities were determined to be present (Fig. 3), and these data were removed from further time series analyses. Inspection of recurrence plots for the remaining four variables (ankle angle, ankle $Y$, knee angle, knee $Y$ ) provided evidence that

TABLE I. Results of standard gait kinematic analysis for three walking conditions.

\begin{tabular}{|c|c|c|c|c|c|c|c|c|}
\hline & \multicolumn{2}{|c|}{ Solo condition } & \multicolumn{3}{|c|}{ Paired condition } & \multicolumn{3}{|c|}{ Forced condition } \\
\hline & Mean & $\mathrm{SD}$ & Mean & SD & $\%$ change & Mean & $\mathrm{SD}$ & $\%$ change \\
\hline Step height (mm) & $\begin{array}{l}166.86 \\
(14.01)\end{array}$ & $\begin{array}{l}16.16 \\
(2.94)\end{array}$ & $\begin{array}{l}168.05 \\
(14.43)\end{array}$ & $\begin{array}{l}17.70 \\
(2.82)\end{array}$ & $\begin{array}{c}4.48 \\
(8.86)\end{array}$ & $\begin{array}{l}167.69 \\
(13.52)\end{array}$ & $\begin{array}{l}17.25 \\
(1.57)\end{array}$ & $\begin{array}{c}5.11 \\
(7.64)\end{array}$ \\
\hline Step length (mm) & $\begin{array}{l}653.95 \\
(32.77)\end{array}$ & $\begin{array}{l}61.36 \\
(7.33)\end{array}$ & $\begin{array}{l}657.66 \\
(67.57)\end{array}$ & $\begin{array}{l}67.57^{\mathrm{a}} \\
(6.02)\end{array}$ & $\begin{array}{c}1.67 \\
(1.15)\end{array}$ & $\begin{array}{l}640.27 \\
(35.91)\end{array}$ & $\begin{array}{l}65.49^{\mathrm{a}} \\
(5.86)\end{array}$ & $\begin{array}{c}3.80 \\
(3.35)\end{array}$ \\
\hline Ankle angle (deg) & $\begin{array}{c}35.60 \\
(4.75)\end{array}$ & $\begin{array}{c}4.20 \\
(0.82)\end{array}$ & $\begin{array}{c}36.53 \\
(5.49)\end{array}$ & $\begin{array}{l}4.64^{\mathrm{a}} \\
(0.91)\end{array}$ & $\begin{array}{c}3.45 \\
(2.96)\end{array}$ & $\begin{array}{c}35.91 \\
(5.84)\end{array}$ & $\begin{array}{c}4.39 \\
(0.91)\end{array}$ & $\begin{array}{c}5.52 \\
(0.04)\end{array}$ \\
\hline Knee angle (deg) & $\begin{array}{l}56.71 \\
(4.53)\end{array}$ & $\begin{array}{c}5.43 \\
(0.54)\end{array}$ & $\begin{array}{l}57.06 \\
(4.55)\end{array}$ & $\begin{array}{l}5.97^{\mathrm{a}} \\
(0.48)\end{array}$ & $\begin{array}{c}2.23 \\
(1.82)\end{array}$ & $\begin{array}{l}56.83 \\
(4.71)\end{array}$ & $\begin{array}{c}5.86 \\
(0.40)\end{array}$ & $\begin{array}{c}3.41 \\
(2.98)\end{array}$ \\
\hline
\end{tabular}

${ }^{\mathrm{a}}$ Significantly different from solo condition at $p<0.05, \%$ change: Normalized absolute value of difference from solo condition. Data are reported as mean (standard deviation). 
TABLE II. Mean estimates of maximal Lyapunov exponents for knee and ankle sagittal plane kinematics.

\begin{tabular}{|c|c|c|c|c|c|c|c|c|}
\hline \multirow[b]{2}{*}{ Variable } & \multicolumn{2}{|c|}{ Solo condition } & \multicolumn{2}{|c|}{ Paired condition } & \multicolumn{2}{|c|}{ Forced condition } & \multicolumn{2}{|c|}{ Surrogate data } \\
\hline & $\lambda_{\text {Short }}$ & $\lambda_{\text {Long }}$ & $\lambda_{\text {Short }}$ & $\lambda_{\text {Long }}$ & $\lambda_{\text {Short }}$ & $\lambda_{\text {Long }}$ & $\lambda_{\text {Short }}$ & $\lambda_{\text {Long }}$ \\
\hline $\begin{array}{l}\text { Ankle } \\
\text { angle }\end{array}$ & $\begin{array}{c}0.655 \\
(0.058)\end{array}$ & $\begin{array}{c}0.070 \\
(0.015)\end{array}$ & $\begin{array}{l}0.658^{\mathrm{a}} \\
(0.067)\end{array}$ & $\begin{array}{l}0.078^{a}, \\
(0.013)\end{array}$ & $\begin{array}{l}0.667^{\mathrm{a}} \\
(0.057)\end{array}$ & $\begin{array}{c}0.068 \\
(0.011)\end{array}$ & $\begin{array}{l}0.237^{\mathrm{a}} \\
(0.053)\end{array}$ & $\begin{array}{l}0.033^{\mathrm{a}} \\
(0.009)\end{array}$ \\
\hline Ankle $Y$ & $\begin{array}{c}0.974 \\
(0.076)\end{array}$ & $\begin{array}{c}0.090 \\
(0.016)\end{array}$ & $\begin{array}{c}1.025 \\
(0.060)\end{array}$ & $\begin{array}{l}0.103^{\mathrm{a}} \\
(0.015)\end{array}$ & $\begin{array}{c}1.029 \\
(0.068)\end{array}$ & $\begin{array}{c}0.088 \\
(0.020)\end{array}$ & $\begin{array}{l}0.559^{\mathrm{a}} \\
(0.096)\end{array}$ & $\begin{array}{l}0.077^{\mathrm{a}} \\
(0.013)\end{array}$ \\
\hline $\begin{array}{l}\text { Knee } \\
\text { angle }\end{array}$ & $\begin{array}{c}0.860 \\
(0.099)\end{array}$ & $\begin{array}{c}0.087 \\
(0.014)\end{array}$ & $\begin{array}{c}0.882 \\
(0.066)\end{array}$ & $\begin{array}{c}0.098^{\mathrm{a}} \\
(0.014)\end{array}$ & $\begin{array}{c}0.886 \\
(0.079)\end{array}$ & $\begin{array}{c}0.085 \\
(0.022)\end{array}$ & $\begin{array}{c}0.546^{\mathrm{a}} \\
(0.120)\end{array}$ & $\begin{array}{c}0.078^{\mathrm{a}} \\
(0.016)\end{array}$ \\
\hline Knee $Y$ & $\begin{array}{c}0.932 \\
(0.102)\end{array}$ & $\begin{array}{c}0.068 \\
(0.014)\end{array}$ & $\begin{array}{c}0.955 \\
(0.071)\end{array}$ & $\begin{array}{l}0.074^{a} b \\
(0.013)\end{array}$ & $\begin{array}{c}0.971 \\
(0.067)\end{array}$ & $\begin{array}{c}0.067 \\
(0.021)\end{array}$ & $\begin{array}{l}0.518^{\mathrm{a}} \\
(0.094)\end{array}$ & $\begin{array}{l}0.046^{\mathrm{a}} \\
(0.023)\end{array}$ \\
\hline
\end{tabular}

${ }^{\mathrm{a}}$ Significantly different from solo condition at $p<0.05$.

${ }^{\mathrm{b}}$ Significantly different from forced condition at $p<0.05$. Data are reported as mean (standard deviation).

each time series was relatively stationary and deterministic (Fig. 3). Results of an additional test for determinism ${ }^{46}$ were relatively consistent among the four remaining variables, ranging from an average of $0.951 \pm 0.021$ for ankle angle to $0.996 \pm 0.001$ for ankle $Y$, where a value of 0 indicates a completely random system and a value of 1 denotes a purely deterministic system.

Figure 4 depicts a sample three-dimensional state space for knee angle under the three walking conditions studied. All estimates of maximal Lyapunov exponents for the reconstructed state spaces were positive, which is generally considered to be a characteristic of time series data that are generated by a chaotic system. Estimates of short term maximal Lyapunov exponents $\left(\lambda_{\text {short }}\right)$ differed significantly between the solo condition and the paired condition, and between the solo condition and the forced condition for ankle angle $(p<0.001$ for both, Table II). Estimates of long term maximal Lyapunov exponents $\left(\lambda_{\text {long }}\right)$ differed significantly between the solo condition and the paired condition in all variables analyzed (Table II). No differences in $\lambda_{\text {long }}$ were detected between the solo condition and the forced condition. In addition, no statistical relationship was found between $\lambda_{\text {short }}$ and $\lambda_{\text {long }}$ and normalized changes in mean step length, step height, knee angle, or ankle angle. Further, no statistical relationship was found between changes in standard deviation and $\lambda_{\text {short }}$ and $\lambda_{\text {long. }}$. Estimates of surrogate data $\lambda_{\text {short }}$ and $\lambda_{\text {long }}$ were significantly lower than those found for actual time series data $(p<0.01$ for all variables), demonstrating that estimates of maximal Lyapunov exponents can be effectively used to distinguish phase randomized data from the original time series data. This result is consistent with previous work of a similar nature. ${ }^{27,28}$

Mean divergence curves are presented in Fig. 5. The average magnitude of divergence can be estimated by the value at which the curve settles. Although the slopes were consistently less for the surrogate data in each case (i.e., $\lambda_{\text {short }}$ and $\lambda_{\text {long }}$ ), the magnitude of divergence for the surrogate data was consistently equal or greater for each variable. In addition, the solo condition resulted in a magnitude of divergence that was consistently less than that of either the paired or forced condition for all variables. Further, the paired walking condition consistently resulted in divergence curves that saturated at a decreased rate when compared to the other walking conditions. Overall, these curves support the idea that walking is not entirely chaotic, as divergence rates were estimated from areas of the curve that were relatively nonlinear. Previous research has indicated that normal bipedal walking in humans, at times, demonstrates signatures
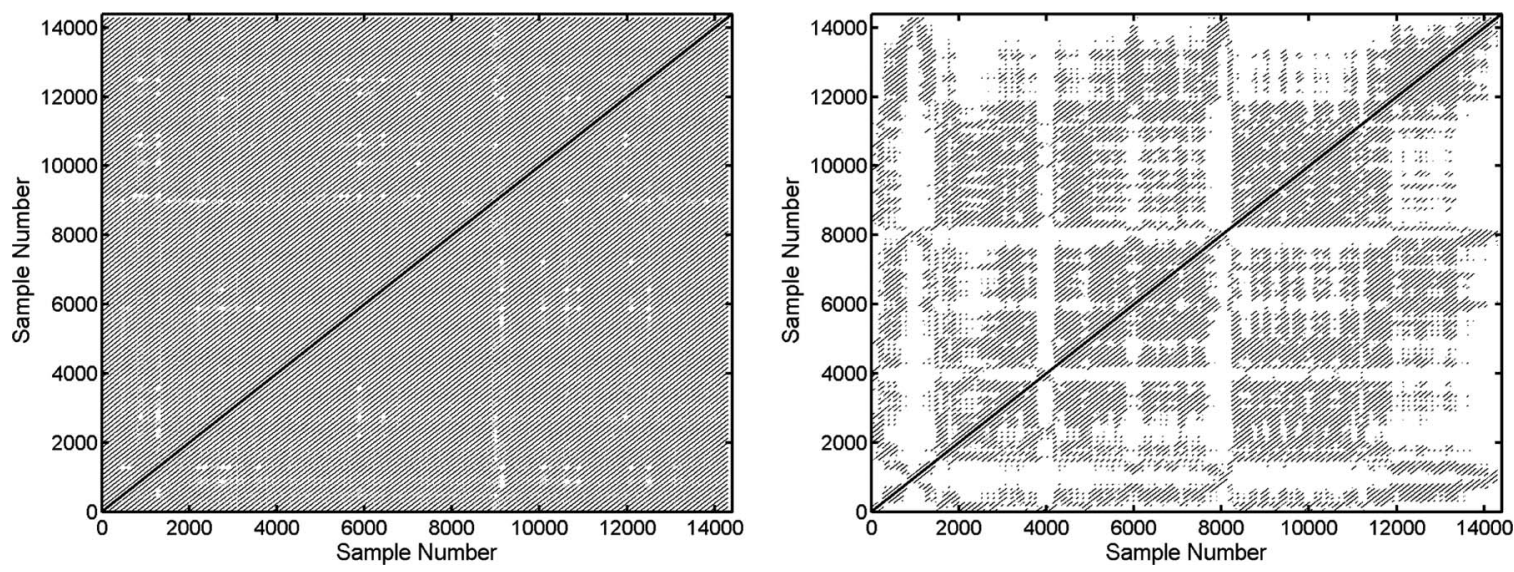

FIG. 3. Sample recurrence plots for ankle $Y$ (left) and ankle $X$ (right). The presence of gross inconsistencies in texture for ankle $X$ indicate nonstationarity. Ankles $X$ and $Z$ data were left out of the nonlinear time series analysis. 

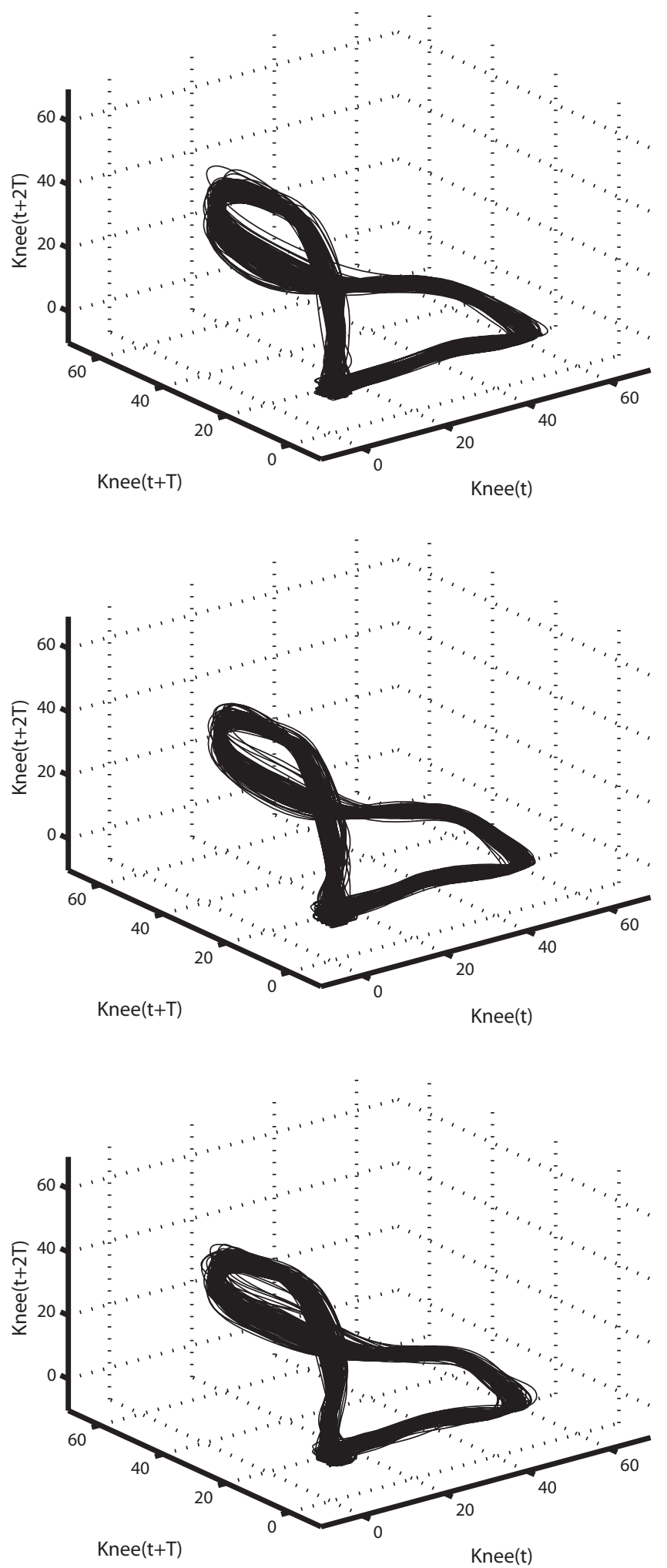

FIG. 4. Three-dimensional state space plots for one subject's knee angle during the solo walking condition (top), paired condition (middle), and forced condition (bottom). An embedding delay of 20 was used.

of chaotic behavior, but more often than not does not fit the definition of a chaotic system. ${ }^{27,29}$ However, distinctly linear regions were observed to occur intermittently for four subjects in the present data, an example of which is illustrated in Fig. 6.

\section{DISCUSSION}

The primary results of this study were twofold. First, the paired walking condition induced involuntary synchronization of stepping that resulted in estimates of stride to stride variability (maximal finite-time Lyapunov exponents) that were significantly increased when compared to the solo and forced walking conditions (Table II). Second, the paired condition also resulted in a significant increase in the standard deviation of knee and ankle sagittal plane kinematics for each individual when compared to either the solo or forced condition (Table I). Estimates of maximal Lyapunov exponents were also significantly lower for phase randomized surrogate data when compared to original time series data and demonstrated a greater magnitude of nearest neighbor divergence (Fig. 5). No correlations were found between standard deviation and estimates of maximal Lyapunov exponents in all cases for any of the variables studied $\left(0.001<r^{2}\right.$ $<0.006)$.

\section{A. Unintentional versus forced synchronization of movement}

Synchronization of movement occurred involuntarily during the paired walking condition, a phenomenon that has been shown to occur previously. ${ }^{8,9,11}$ This simple idea suggests that sensory information regarding the movement of one's partner results in a subconscious, self-organization of entrained movement patterns, and likely involves the activity of mirror-neuron networks that play a role in movement imitation. This condition is fundamentally different from the forced condition where the intention to synchronize involves additional volitional control which can affect motor output. Previous investigators have demonstrated that an increase in intentional control of a movement typically results in greater variability and is most often utilized during the early stages of skill acquisition. Conversely, a reduction in intentional control and a greater reliance on the natural dynamics of the movement lead to decreased variability and are the hallmark of expertise (e.g., Refs. 18-20). The subjects involved in this study likely had little experience synchronizing their stepping with a partner while walking on side by side treadmills, hence it was expected that variability would increase for the forced condition. In most cases, however, there was little difference in variability in performance when the forced condition was compared to either the solo or paired condition, suggesting that this trend may not hold true when a healthy individual performs a relatively unpracticed variation of an over-learned motor skill.

\section{B. Increased kinematic variability during passive side by side walking}

The relationship between maximal Lyapunov exponents, locomotor performance, and the overall health of a system remains relatively unclear. Determining this relationship is further complicated by the presence of stochastic noise, multiple attractors, and time delays in the dynamical system. Typically, estimates of maximal Lyapunov exponents have been interpreted as measures of how the locomotor system responds to minute perturbations over a period of multiple 

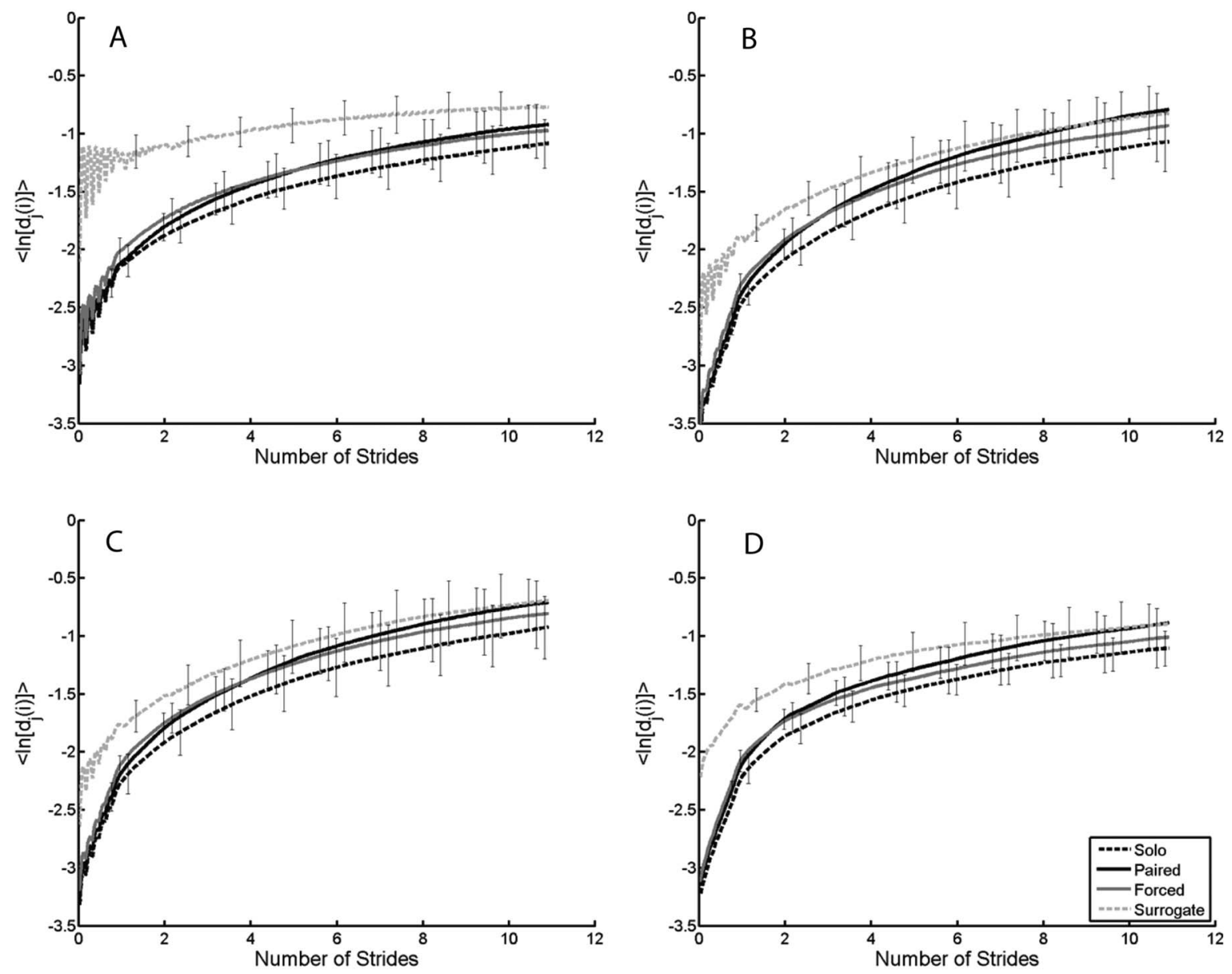

FIG. 5. Mean divergence curves for the solo walking condition, paired condition, forced condition, and surrogate data for ankle angle (a), ankle $Y$ (b), knee angle (c), and knee $Y(\mathrm{~d})$. Bars represent standard deviation $(n=14$ for each condition).

strides. ${ }^{27-29,31,53}$ This idea holds that the natural stride to stride fluctuations in the output of the locomotor system, such as small changes in step length or height, can be viewed as a type of local perturbation that contributes to the aperiodic nature of bipedal walking. This type of analysis therefore provides insight to how individual variations in locomo-

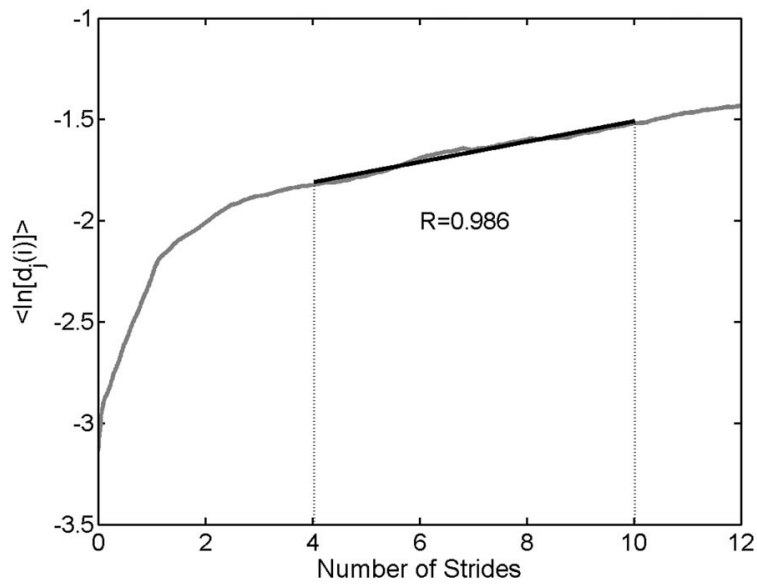

FIG. 6. Sample data for an individual who demonstrated signatures of chaotic behavior during treadmill walking for ankle $Y$ data. The presence of a nearly linear region is uncommon in gait data and has led previous authors to suggest that bipedal walking is not inherently chaotic. tor dynamics are interrelated among multiple, consecutive strides. However, previous investigators have also used estimates of maximal Lyapunov exponents to infer changes in the local stability of a kinematic variable, ${ }^{27-29,31}$ a relationship that is not well defined. While the response of a system to local perturbations can provide insight to the nature of the motor output, it is unclear how changes in kinematic variability reflect changes in the stability of walking. Instead, changes in maximal Lyapunov exponents might be interpreted more appropriately as alterations to the locomotor attractor itself. For example, using the illustration of a marble rolling over a manifold, increased variability of the performance of a motor skill might indicate a change to the depth of the well or fixed-point attractor, or as a change in the "steepness" of its walls. ${ }^{18}$ Figure 5 demonstrates a decreased rate of saturation for the paired condition when compared with the forced and solo conditions. This could imply a reduction in the steepness of the walls of the locomotor attractor, as local perturbations affected the locomotor system for longer periods of time (i.e., over more strides) when subjects were synchronized involuntarily.

A second but similar interpretation of these results can be described as a basin of neighboring fixed-point attractors that experiences an increase in interattractor maneuverability. During the paired walking condition, most couples were not 
synchronized over the entire 2 min period analyzed (although all were synchronized for at least $1 \mathrm{~min}$ ). Therefore, the current results might also be explained as an "engagement" and "disengagement" from the mutual stepping pattern that was negotiated between each subject pair. An increase in stride to stride variability might therefore arise as the result of a subject moving between two different attractors, one based on their preferred walking pattern and a second based on the synchronized stepping pattern. In this context, variability might also be increased by the uncertain and evolving nature of the second attractor, which is based on the negotiated stepping pattern. This negotiation is likely a continual process, whereby each partner repeatedly attempts to pull the negotiated pattern closer to their preferred pattern and might therefore alter the attributes of the synchronized attractor with each step.

A third interpretation might also be considered when these results are compared with those of previous research that has observed alterations in kinematic variability during gait in aged and diseased individuals. ${ }^{14,27-29,32}$ The size of the locomotor basin of attraction may be related to the number of movement patterns, or fixed-point attractors, available to an individual. ${ }^{30,39}$ In this context, the amount of stride to stride variability measured for a particular joint would reflect the number of stable locomotor movement patterns that are used by an individual in a given situation. A locomotor system might therefore be characterized by the size of its "library" or the number of stable movement patterns available and how those movement patterns are accessed and utilized during terrestrial locomotion. A healthy individual would likely posses a large number of possible movement patterns but would be expected to utilize relatively few while walking under normal conditions. When walking stability is challenged, the healthy individual can then select from many potential movement options in order to avoid falling. Research in passive dynamic walking models suggests that this basin of attraction can be further widened by including chaotic walking patterns that provide stability over a limited number of steps to serve as a transitional state for an individual to return to a more deterministic walking pattern. ${ }^{30,39}$ Based on the present results, side by side walking would be an example of a condition in which additional, stable locomotor patterns are accessed and utilized by a healthy individual. Individuals with increased age illustrate a different aspect of this idea by demonstrating increased variability in lower-limb movement kinematics under normal circumstances. ${ }^{28,29}$ This result may suggest that physiological changes as a result of normal aging require that an individual utilize an increased number of possible movement patterns when the system is relatively unchallenged. Such increased flexibility or maneuverability within the basin of attraction might be detrimental for a couple of reasons. First, it may lead to an individual spending increased time utilizing movement patterns that are near the boundary of the basin, where a relatively small perturbation might more easily push them into instability. Second, an individual that uses a larger proportion of their stable gait patterns may also have fewer options remaining when perturbed, leading to an increase in fall risk. In the case of an injured or neurologically impaired individual, variability may actually be decreased in certain cases $^{34,36}$ due in part to a decrease in the size of their library or overall number of stable movement patterns (i.e., fixedpoint attractors) available.

\section{Side by side walking as a therapeutic technique}

Paired walking in which an unhealthy individual walks side by side with a healthy individual may be an effective practice for the restoration of locomotor function. Previous experiments have successfully utilized external cues as an artificial pacemaker for the purpose of improving locomotor function in neurologically impaired individuals. ${ }^{5,6}$ Hausdorff et al., ${ }^{7,13}$ however, presented evidence that forced walking to the auditory output of a metronome resulted in a decrease in the stability of long range correlations in stride time that are normally seen in healthy individuals. Taken together, these results suggest that some type of synchronization with an external cue can be beneficial for rehabilitation, but an auditory cue that is presented at a constant frequency may not be the optimal use of this technique. Side by side walking might be a more effective approach for several reasons. First, a healthy locomotor system can be used to generate a synchronizing signal that involves both a biologically varying cadence and optimal variability in joint kinematics. Second, synchronization can be achieved unintentionally, which is more analogous to normal walking conditions, and limits the use of intentional, corrective movements that are often related to early stages of skill acquisition. ${ }^{18-20}$ Third, visual and auditory information regarding a partner's movements stimulates an inherent tendency to synchronize, possibly using neuronal networks that are predisposed to imitating human motion as a part of the motor learning process. ${ }^{22-24}$ Fourth, the present results demonstrate that side by side walking results in an increase in stride to stride variability, which suggests that this approach can be used to effectively manipulate the stride to stride dynamics of gait. Finally, there is evidence to suggest that a form of social memory may persist for this type of interaction. ${ }^{3}$ In other words, following interpersonal synchronization of movement, one's preferred movement pattern remains altered for a time, suggesting that more permanent alterations in an abnormal basin of attraction for locomotion might be achieved by unintentionally synchronized walking.

Further research is necessary to determine if the use of side by side walking is an effective method to achieve gains in locomotor function for impaired individuals. Specifically, it remains to be seen if side by side walking, where the external signal is adaptable and time variant, will produce results different from those reported by Hausdorff et al. ${ }^{7}$ and whether this type of practice will yield clinical results. ${ }^{5,6}$ The current results are an initial step in that direction by providing evidence to suggest that walking side by side will result in a different outcome when compared with therapeutic techniques that require a patient to walk independently. In addition to side by side walking, this effect might also be reproduced in a clinic using virtual reality, video, mechanical input, or an auditory recording of an unimpaired person stepping. 
Finally, several investigators have previously demonstrated differences in lower-limb kinematics when treadmill walking was compared to walking over ground. ${ }^{54-56}$ In particular, Dingwell and Cusumano ${ }^{27,31}$ reported significant reductions in estimates of maximal Lyapunov exponents for treadmill walking when compared to overground walking for several kinematic variables. Overall, there appears to be a general consensus that treadmill walking artificially decreases variability of movement kinematics. While the use of motorized treadmills in the present study was a factor in the behavior of the variables analyzed, it should be noted that this factor was applied to all subjects equally and would likely only serve to reduce the observed effect of increased stride to stride variability during the paired condition. In the future, it would be useful to perform a similar analysis during overground walking, where differences in variability may possibly be more dramatic.

${ }^{1}$ M. J. Richardson, K. L. Marsh, R. W. Isenhower, J. R. L. Goodman, and R. C. Schmidt, "Rocking together, dynamics of intentional and unintentional interpersonal coordination," Hum. Mov. Sci. 26, 867 (2007).

${ }^{2}$ M. J. Richardson, K. L. Marsh, and R. C. Schmidt, "Effects of visual and verbal interaction on unintentional interpersonal coordination," J. Exp. Psychol. Hum. Percept. Perform. 31, 62 (2005).

${ }^{3}$ O. Oullier, G. C. de Guzman, K. J. Jantzen, J. Lagarde, and J. A. S. Kelso, "Social coordination dynamics: Measuring human bonding," Soc. Neurosci. Symp. 3, 178 (2008).

${ }^{4}$ R. C. Schmidt and M. T. Turvey, "Phase-entrainment dynamics of visually coupled rhythmic movements," Biol. Cybern. 70, 369 (1994).

${ }^{5}$ M. H. Thaut, G. C. McIntosh, R. R. Rice, R. A. Miller, J. Rathbun, and J. M. Brault, "Rhythmic auditory stimulation in gait training for Parkinson's disease patients," Mov. Disord. 11, 193 (1996).

${ }^{6}$ M. Roerdink, C. J. C. Lamoth, G. Kwakkel, P. C. W. van Wieringen, and P. J. Beek, "Gait coordination after stroke: Benefits of acoustically paced treadmill walking," Phys. Ther. 87, 1010 (2007).

${ }^{7}$ J. M. Hausdorff, P. L. Purdon, C. K. Peng, Z. Ladin, J. Y. Wei, and A. L. Goldberger, "Fractal dynamics of human gait: Stability of long-range correlations in stride interval fluctuations," J. Appl. Physiol. 80, 1448 (1996). ${ }^{8}$ A. Z. Zivotofsky and J. M. Hausdorff, "The sensory feedback mechanisms enabling couples to walk synchronously: An initial investigation," J. Neuroeng. Rehabil. 4, 28 (2007).

${ }^{9}$ N. R. van Ulzen, C. J. C. Lamoth, A. Daffertshofer, G. R. Semin, and P. J. Beek, "Characteristics of instructed and uninstructed interpersonal coordination while walking side by side," Neurosci. Lett. 432, 88 (2008).

${ }^{10}$ S. H. Strogatz, D. M. Abrams, A. McRobie, B. Eckhardt, and E. Ott, "Theoretical mechanics: Crowd synchrony on the Millennium Bridge," Nature (London) 438, 43 (2005).

${ }^{11}$ J. A. Nessler and S. J. Gilliland, "Interpersonal synchronization during side by side treadmill walking is influenced by leg length differential and altered sensory feedback," Hum. Mov. Sci. (in press).

${ }^{12}$ J. M. Hausdorff, J. Lowenthal, T. Herman, L. Gruendlinger, C. Peretz, and N. Giladi, "Rhythmic auditory stimulation modulates gait variability in Parkinson's disease," Eur. J. Neurosci. 26, 2369 (2007).

${ }^{13}$ J. M. Hausdorff, S. L. Mitchell, R. Firtion, C. K. Peng, M. E. Cudkowicz, J. Y. Wei, and A. L. Goldberger, "Altered fractal dynamics of gait: Reduced stride-interval correlations with aging and Huntington's disease," J. Appl. Physiol. 82, 262 (1997).

${ }^{14}$ J. M. Hausdorff, H. K. Edelberg, S. L. Mitchell, A. L. Goldberger, and J. Y. Wei, "Increased gait unsteadiness in community dwelling elderly fallers," Arch. Phys. Med. Rehabil. 78, 278 (1997).

${ }^{15}$ P. E. Rapp, I. D. Zimmerman, A. M. Albano, G. C. de Guzman, N. N. Greenbaun, and T. R. Bashore, "Experimental studies of chaotic neural behavior: Cellular activity and electroencephalographic signals," in Nonlinear Oscillations in Biology and Chemistry (Springer-Verlag, Berlin, Germany, 1986), pp. 175-205.

${ }^{16}$ A. L. Goldberger, D. R. Rigney, J. Mietus, E. M. Antman, and S. Greenwald, "Nonlinear dynamics in sudden cardiac death syndrome: Heart rate oscillations and bifurcations," Experientia 41, 983 (1998).
${ }^{17}$ A. L. Goldberger, V. Bhargava, B. West, and A. J. Mandell, "Some observations on the question: Is ventricular fibrillation "chaos?"” Physica D 19, 282 (1986).

${ }^{18}$ J. G. Milton, S. S. Small, and A. Solodkin, "On the road to automatic: Dynamic aspects in the development of expertise," J. Clin. Neurophysiol. 21, 134 (2004).

${ }^{19}$ P. J. Beek and M. T. Turvey, "Temporal patterning in cascade juggling," J. Exp. Psychol. Hum. Percept. Perform. 18, 934 (1992).

${ }^{20}$ J. L. Cabrera and J. G. Milton, "Human stick balancing: Tuning Levy flights to improve balance and control," Chaos 14, 691 (2004).

${ }^{21}$ S. J. Gilliland and J. A. Nessler, "Entrainment during side by side treadmill walking is influenced by interpersonal leg length difference," in Annual Meeting American Physical Therapy Association, Baltimore, MD, 2009 (unpublished).

${ }^{22}$ S. Cochin, C. Barthelemy, S. Roux, and J. Martineau, "Observation and execution of movement: Similarities demonstrated by quantified electroencephalography," Eur. J. Neurosci. 11, 1839 (1999).

${ }^{23}$ E. Kohler, C. Keysers, M. A. Umilta, L. Fogassi, V. Gallese, and G. Rizzolatti, "Hearing sounds, understanding actions: Action representation in mirror neurons," Science 297, 846 (2002).

${ }^{24}$ G. Rizzolatti and L. Craighero, "The mirror-neuron system," Annu. Rev. Neurosci. 27, 169 (2004)

${ }^{25}$ J. Issartel, L. Marin, and M. Cadopi, "Unintended interpersonal coordination: 'Can we march to the beat of our own drum?'” Neurosci. Lett. 411, 174 (2007).

${ }^{26}$ J. A. S. Kelso, The Self-Organization of Brain and Behavior (MIT, Cambridge, MA, 1995).

${ }^{27}$ J. B. Dingwell and J. P. Cusumano, "Nonlinear time series analysis of normal and pathological human walking," Chaos 10, 848 (2000).

${ }^{28}$ U. H. Buzzi, N. Stergiou, M. J. Kurz, P. A. Hageman, and J. Heidel, "Nonlinear dynamics indicates aging affects variability during gait," Clin. Biomech. (Bristol, Avon) 18, 435 (2003).

${ }^{29}$ H. G. Kang and J. B. Dingwell, "Effects of walking speed, strength, and range of motion on gait stability in healthy older adults," J. Biomech. 41, 2899 (2008).

${ }^{30}$ M. J. Kurz and N. Stergiou, "Do horizontal propulsive forces influence the nonlinear structure of locomotion?" J. Neuroeng. Rehabil. 4, 30 (2007).

${ }^{31}$ J. B. Dingwell, J. P. Cusumano, P. R. Cavanagh, and D. Sternad, "Local dynamic stability versus kinematic variability of continuous overground and treadmill walking," J. Biomech. Eng. 123, 27 (2001).

${ }^{32}$ J. M. Hausdorff, "Gait variability: Methods, modeling and meaning," J. Neuroeng. Rehabil. 2, 19 (2005).

${ }^{33}$ Y. Ohtaki, M. Arif, A. Suzuki, K. Fujita, H. Inooka, R. Nagatomi, and I. Tsuji, "Assessment of walking stability of elderly by means of nonlinear time series analysis and simple accelerometry," JSME Int. J., Ser. C 48 607 (2005).

${ }^{34}$ R. E. van Emmerik, R. C. Wagenaar, A. Winogrodzka, and E. C. Wolters, "Identification of axial rigidity during locomotion in Parkinson's disease," Arch. Phys. Med. Rehabil. 80, 186 (1999).

${ }^{35}$ B. C. Heiderscheit, "Movement variability as a clinical measure for locomotion," J. Appl. Biomech. 16, 419 (2000).

${ }^{36}$ J. Hamill, R. E. van Emmerik, B. C. Heiderscheit, and L. L. Li, "A dynamical systems approach to lower extremity running injuries," Clin. Biomech. (Bristol, Avon) 14, 297 (1999).

${ }^{37}$ J. M. Hausdorff, "Gait dynamics, fractals and falls: Finding meaning in the stride to stride fluctuations of human walking," Hum. Mov. Sci. 26, 555 (2007).

${ }^{38}$ O. Beauchet, G. Allali, G. Berrut, and V. Dubost, "Is lower limb kinematic variability always an index of stability?" Gait and Posture 26, 327 (2007).

${ }^{39}$ M. J. Kurz, T. N. Judkins, C. Arellano, and M. Scott-Pandorf, "A passive dynamic walking robot that has a determininstic nonlinear gait," J. Biomech. 41, 1310 (2008).

${ }^{40}$ R. Hegger, H. Kantz, and T. Schreiber, "Practical implementation of nonlinear time series methods: The TISEAN package," Chaos 9, 413 (1999).

${ }^{41}$ M. Perc, "The dynamics of human gait," Eur. J. Phys. 26, 525 (2005).

${ }^{42}$ A. M. Fraser and H. L. Swinney, "Independent coordinates for strange attractors from mutual information," Phys. Rev. A 33, 1134 (1986).

${ }^{43}$ H. D. I. Abarbanel, Analysis of Observed Chaotic Data (Springer, New York, 1996).

${ }^{44}$ M. B. Kennel, R. Brown, and H. D. I. Abarbanel, "Determining embedding dimension for phase-space reconstruction using a geometrical construction," Phys. Rev. A 45, 3403 (1992). 
${ }^{45}$ J. P. Eckmann, S. O. Kamphorst, and D. Ruelle, "Recurrence plots of dynamical systems," Europhys. Lett. 4, 973 (1987).

${ }^{46}$ D. Kaplan and L. Glass, Understanding Nonlinear Dynamics (SpringerVerlag, New York, 1995).

${ }^{47} \mathrm{H}$. Kantz, "A robust method to estimate the maximal Lyapunov exponent of a time series," Phys. Lett. A 185, 77 (1994).

${ }^{48}$ M. T. Rosenstein, J. J. Collins, and C. J. De Luca "A practical method for calculating largest Lyapunov exponents from small data sets," Physica D 65, 117 (1993)

${ }^{49}$ J. Theiler, "Estimating fractal dimension," J. Opt. Soc. Am. A 7, 1055 (1990).

${ }^{50} \mathrm{P}$. Pascolo, F. Barazza, and R. Carniel, "Considerations on the application of the chaos paradigm to describe the postural sway," Chaos, Solitons Fractals 27, 1339 (2006).

${ }^{51}$ B. Manor, P. Wolenski, and L. Li, "Faster walking speeds increase local instability among people with peripheral neuropathy," J. Biomech. 41, 2787 (2008)

${ }^{52} \mathrm{H}$. Kantz and T. Schreiber, Nonlinear Time Series Analysis, 2nd ed. (Cambridge University Press, Cambridge, 2004).

${ }^{53}$ S. A. England and K. P. Granata, "The influence of gait speed on local dynamic stability of walking," Gait and Posture 25, 172 (2007).

${ }^{54}$ V. Wank, U. Frick, and D. Schmidtbleicher, "Kinematics and electromyography of lower limb muscles in overground and treadmill running," Int. J. Sports Med. 19, 455 (1998).

${ }^{55}$ M. E. Pearce, D. A. Cunningham, A. P. Donner, P. A. Rechnitzer, G. M. Fullerton, and J. H. Howard, "Energy cost of treadmill and floor walking at self-selected paces," Eur. J. Phys. 52, 115 (1983).

${ }^{56}$ S. C. White, H. J. Yack, C. A. Tucker, and H. Y. Lin, "Comparison of vertical ground reaction forces during overground and treadmill walking," Med. Sci. Sports Exercise 30, 1537 (1998). 
Chaos is copyrighted by the American Institute of Physics (AIP). Redistribution of journal material is subject to the AIP online journal license and/or AIP copyright. For more information, see http://ojps.aip.org/chaos/chocr.jsp 